\title{
Commentary: micromanaging oxidative stress: how gametes and embryos survive in their cozy niches!
}

\author{
David F. Albertini
}

Published online: 13 September 2014

(C) Springer Science+Business Media New York 2014

On Planet Earth, the emergence of eukaryotic life forms in an oxygen rich atmosphere came with a cost. As metabolic pathways evolved to meet the growing bioenergetic demands of more sophisticated growth and survival activities, provisioning sufficient quantities of ATP required the oxidative phosphorylation capacity engendered in mitochondria. Although students of evolutionary biology still debate how mitochondria assumed their appointed place in a eukaryotic landscape, uncontestable is the significance of the proton pump and electron transport chain of these essential organelles that brought with it the driving force for higher forms of life and their attendant and growing bioenergetic requirements. As is all too well known, a necessary and sufficient byproduct of mitochondrial metabolism is the generation of reactive oxygen species, or ROS. This potentiated array of molecules, which in excess challenge the endogenous reductive potential of cells, represents an immediate and ever-present threat to the stability and function of many a biomolecule. Thus, be it lipids, carbohydrates, proteins, or nucleic acids, oxidative modifications of these entities provide an established basis for many disease states and rests at the heart of many models attempting to explain the progressive loss of cell and tissue function associated with advancing age.

So it is that we have come to appreciate, and in some ways obsess over, this fundamental aspect of eukaryotic cell biology in the context of human reproduction. That the delicate balance between ROS generation and protection of our gametes and embryos through their developmental history is vital to

Capsule A central challenge in the field of human ARTs is to ameliorate the consequences of atmospheric oxygen when gametes and embryos are handled ex vivo. Characterizing the sources and nature of ROS-induced damage will prompt the more widespread use of anti-oxidant therapies for the management of human fertility, in vivo and in vitro.

D. F. Albertini $(\bowtie)$

University of Kansas Medical Center, Kansas, KS, USA

e-mail: dalbertini@kumc.edu their survival would be an understatement in the world of ARTs. After all, the niches deployed for gametogenesis, ovum transport, and implantation share the common feature of outsourcing ROS in the immediate vicinity of cargoes that at one time or another must perform at extreme lengths to protect against oxidative damage and at the same time support heightened levels of metabolism required to sustain critical processes. For example, while Sertoli cells nurture developing sperm, they are at the same time subject to metabolic demands that threaten the livelihood of the male genome as it progresses through meiosis and the protamine-based compaction of chromatin. Similarly, in the ovary, companion granulosa cells serve the gametic cargo well in metabolic support and offer a unique mechanism of future protection by endowing the oocyte with large quantities of the physiological reducing agent glutathione, which will be deployed during male pronucleus formation by protamine-histone exchange and will serve to protect the zygote as it passages through the Fallopian tubes and into the uterine cavity (itself a hotbed of ROS).

How gametes and embryos manage the threat of ROSinduced damage is, then, the focus of this issue of JARG. Launching our coverage of this seasoned and persistent problem in human ARTs is the paper by Meuter and colleagues from the Mayo Clinic (see cover and Markers of cellular senescence are elevated in murine blastocysts cultured in vitro: molecular consequences of culture in molecular oxygen, DOI 10.1007/s10815-014-0299-8). Among the targets of ROS-induced damage in gametes and embryos is the DNA itself. Double-strand breaks (DSBs) in DNA are but one type of lesion caused by free radicals, chemotherapy, or radiation. In all of these cases, cells respond to DSBs by activating an ATM-based signaling cascade that results in the phosphorylation of the histone variant $\mathrm{H} 2 \mathrm{AX}$, an intermediate in what is referred to as the DNA Damage and Repair Response (DDR). While many adverse consequences of embryo culture in atmospheric oxygen $(20 \%)$ have been 
recorded including those involving epigenetic modifications known to impact later development and offspring health, whether such conditions create an aging phenotype in the embryo has not been directly addressed. The present study demonstrates an increased incidence of DSBs, a biomarker of aging in many cell types, under conditions of atmospheric oxygen, which interestingly, can be minimized when mouse embryos are cultured under low oxygen tension. That the trend in human ARTs is to reduce oxygen tension for embryo culture is heartening in this light especially with the move to extended culture to the blastocyst stage that offers a greater degree of assessment of other parameters of embryo quality afforded by PGS. But concerns over ROS are not limited to the embryo!

What about those precious and determinative phases of gametogenesis when oocytes and sperm are harbored, respectively, in the follicle or seminiferous tubule? Two new studies focus on gamete quality and somatic contributions to the microenvironment.

Gourabi and colleagues report on the consequences of treating male mice with a potent oxidant that tips the balance of ROS production over that of the ability to generate enough antioxidant in the form of reduced glutathione so as to produce an intratesticular oxidative stress situation (TBHP-induced oxidative stress alters microRNAs expression in the mouse testis, DOI 10.1007/s10815-014-0302-4). They document the kinetics of this effect and note in particular lesions in spermatogenesis at the Sertoli cell spermatocyte junction while these cells are dealing with the natural rectification of DSBs associated with meiotic homologous recombination-a double whammy, so to speak! And the molecular nature of the damage seems to come at the expense of micro RNAs regulating spermatogenesis.

From Piomboni et al. comes a report on the expression of oxidative stress biomarkers in follicular fluid from PCOS patients who have received supplements of inositol or metformin in management of their disease (Protein modification as oxidative stress markers in follicular fluid from women with polycystic ovary syndrome: the effect of inositol and metformin, DOI 10.1007/s10815-014-0307-z). Finding non- invasive ways to monitor oxidative stress is not easy. Here, in order to obtain a broad stroke image of oxidative status in follicles, fluid samples were analyzed using a seasoned biochemical assay for protein-free sulfhydryl group content that exploits the specificity of fluorescent maleimide derivatives making quantitation possible. As you will see, PCOS patients benefit from metformin and inositol treatments as reflected in lower levels of oxidative stress and an enhancement in oocyte quality and pregnancy rates.

Finally, we draw attention to yet another study in which the potential utility of gene expression profiling of cumulus cells as an indicator of ART outcome is suggested. However, in this case patients with endometriosis are evaluated, and the results are provocative in terms of how inflammatory conditions in the reproductive tract-a state of affairs linked to oxidative stress-may impact the most proximal cellular environment in which oocytes reside (Allegra et al.,The gene expression profile of cumulus cells reveals altered pathways in patients with endometriosis, DOI 10.1007/s10815-014-0305-1). That the textbook molecular description of ovulation as we now know it has been rewritten as an inflammatory explosion comes as no surprise. But in the face of such a risky process reserved to deal with infection comes an even greater need to explain how it is that the oocyte is protected from the perfect storm within which it finds itself. The reader's imagination need not be stretched given the identification of misregulated cumulus genes in patients with endometriosis that goes well beyond the cytokines expected at ovulation and draws attention to potent chemotaxis gene products, like CXCL2, suspected of enhancing leukocyte infiltration to an extent that would not be advisable under normal physiological conditions.

The delicate balance between metabolism and reproductive competence will continue to provide a platform for future developments in human ARTs. Hopefully, this issue brings our readership a step closer to the ways in which our patients and their extricated gametes and embryos can be micromanaged to prevent the unavoidable scourge of ROS and the attendant risks associated with oxidative stress. 